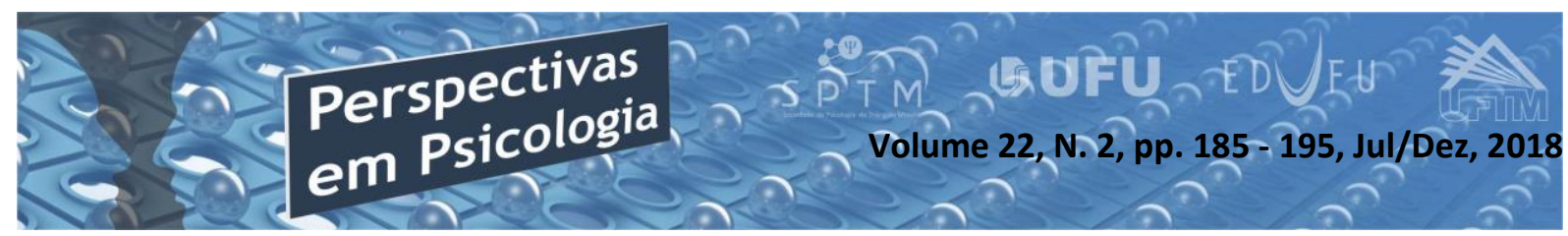

\title{
A ATUAÇÃO DO ESTAGIÁRIO DE PSICOLOGIA: UM RELATO DE EXPERIÊNCIA
}

\author{
Gustavo Souza Zanatta \\ Patricky Batista da Silva \\ Marcelo Augusto Zacarias \\ (Universidade Luterana do Brasil - CEULM/ULBRA, Manaus - AM)
}

\begin{abstract}
Resumo
O presente artigo tem como objetivo relatar as experiências vivenciadas pelos estagiários de psicologia do Centro Universitário Luterano de Manaus - ULBRA, no Ministério Público do Estado do Amazonas, Procuradoria-Geral de Justiça (MPAM-PGJ), em um programa de atenção às vítimas de violência e vulnerabilidade social, situado na cidade de Manaus, Amazonas, no período de novembro de 2017 a maio de 2018, totalizando seis meses. A ideia inicial partiu da disciplina de Estágio Específico em Ênfase, onde a disciplina proporcionou os primeiros atendimentos clínicos de abordagem psicanalítica e supervisão. Todo o processo foi dinâmico e gradativo, com o professor/supervisor auxiliando e sanando as dúvidas. As experiências do estágio no programa proporcionaram aos acadêmicos um espaço de entendimento, aplicação e consolidação dos conhecimentos teóricos, vinculados junto à prática dos atendimentos e supervisões com os psicólogos do programa. Dentro deste contexto foi possível perceber que mesmo sendo um programa desenvolvido no âmbito jurídico para subsidiar o trabalho dos promotores, o psicossocial trabalha visando sempre à pessoa/família atendida como principal foco de emancipação, empoderamento e ressignificação.
\end{abstract}

Palavras-chave: Experiência; estágio; clínica.

\begin{abstract}
The objective of this article is to report on the experiences of psychology trainees at the Lutheran University Center of Manaus - ULBRA, at the Public Prosecutor's Office of the State of Amazonas, Attorney General's Office (MPAM-PGJ). violence and social vulnerability, located in the city of Manaus, Amazonas, from November 2017 to May 2018, totaling six months. The initial idea started from the subject of Specific Stage in Emphasis, where the material provided the first clinical care of a psychoanalytic approach and supervision. The whole process was dynamic and gradual, with the teacher / supervisor assisting and healing the doubts. The internship experiences in the program provided the academics with a space for understanding, applying and consolidating the theoretical knowledge, linked to the practice of attendance and supervision with the Psychologists of the program. Within this context, it was possible to perceive that, even though it is a program developed in the Legal framework to subsidize the work of the promoters, the psychosocial works always aiming at the person / family attended as the main focus of emancipation, empowerment and resignification.
\end{abstract}

Key words: Experience; internship; clinic. 


\section{Introdução}

$\mathrm{O}$ presente artigo trata-se de um relato de experiência enquanto acadêmicos do curso de psicologia do Centro Universitário Luterano de Manaus-ULBRA em um programa de atenção às vítimas de violência, atuando no setor de Psicologia, localizado no município de Manaus, estado do Amazonas, no bairro Aleixo. Tem como objetivo relatar a consolidação da experiência de aproximação da universidade dos serviços públicos de apoio psicossocial e da contribuição da prática psicológica, quanto estagiários de Psicologia.

O trabalho utiliza-se do método de relato de experiência profissional, em que os acadêmicos foram inseridos no contexto exposto e lhes foram designadas tarefas tais como acompanhar visitas domiciliares junto a equipe de psicólogos responsáveis pelos casos, participar dos atendimentos realizados no programa e das reuniões de estudos de casos, apresentar relatório das atividades realizadas, abordando sua atuação, conteúdos aprendidos e impacto da atividade na formação pessoal e acadêmica e realizar, quando solicitado, palestras nas escolas públicas sobre temas inerentes ao programa , tais como: abuso sexual, trabalho infantil, alienação parental, bullying, etc.
O programa tem como objetivo trabalhar com vítimas de violência, segundo Monteiro (2012) um dos objetivos do atendimento psicológico às vítimas é fazer com que elas resgatem sua condição de sujeito, bem como sua autoestima, suas vontades e desejos, que ficaram ignorados e abolidos durante todo o período em que vivenciaram em uma situação traumática marcada pela violência. Dessa forma, a ressignificação é feita de forma gradual para que se obtenha um resultado duradouro.

As atividades apresentadas neste artigo compreendem o período de novembro de 2017 a maio de 2018, em que foi possível acompanhar casos desde que chegaram ao programa até a elaboração dos relatórios. As experiências do estágio no programa proporcionaram aos acadêmicos um espaço de entendimento, aplicação e consolidação dos conhecimentos teóricos, vinculados junto à prática dos atendimentos e supervisões com os psicólogos do programa. Dentro deste contexto, foi possível perceber que mesmo sendo um programa desenvolvido no âmbito jurídico para subsidiar o trabalho dos promotores, $\mathrm{o}$ psicossocial trabalha visando sempre à pessoa/família atendida como principal foco de emancipação, empoderamento e ressignificação.

\subsection{Histórico do Programa}


O programa foi criado no mês de julho de 2016 em uma parceria do Ministério Público do Estado do Amazonas com o Governo do Estado do Amazonas, para oferecer subsídio ao trabalho dos procuradores e promotores de justiça e atuar no atendimento às pessoas em situação de vulnerabilidade psicossocial, bem como no atendimento de demandas das Procuradorias e Promotorias no desenvolvimento das ações ministeriais que visem a garantia dos direitos e garantias fundamentais dos cidadãos e possui como principal objetivo desenvolver um trabalho através de um olhar humanizado às pessoas em situação de vulnerabilidade psicossocial, fornecendo um atendimento acolhedor, de modo a estimular a reintegração social e familiar dessas pessoas, apoiar em suas inciativas em busca de autonomia, contribuindo na promoção do bem-estar e da qualidade de vida, tendo como referência o trabalho em rede intersetorial. Ministério Público do Estado do Amazonas - Procuradoria-Geral de Justiça [MPAM-PGJ] (2016).

Atualmente, o programa é formado por uma equipe interprofissional. Profissionais de diferentes áreas, entre elas, assistentes sociais (2), psicólogos (3), pedagogo (1), agente técnico jurídico (1) e promotor de justiça (1) trabalham juntos, mantendo suas atuações específicas, com troca de informações dentro da área de interseção, juntamente com os estagiários supervisionados sendo três de serviço social, três de psicologia e um de pedagogia. A atuação e serviços oferecidos de cada especialidade, de acordo com MPAMPGJ (2016) são divididas da seguinte forma:

a) Serviço Social - visa promover o acolhimento da pessoa em situação de vulnerabilidade, a identificação de suas expectativas em relação às ações da equipe e o encaminhamento a psicólogo. A proposta é de que a intervenção social seja extensiva aos familiares e/ou colaterais;

b) Psicologia - busca articular estratégias e ações com o intuito de promover a qualidade de vida das pessoas atendidas no programa, buscando reduzir os fatores de risco psicossocial e fortalecer os fatores de proteção;

c) Orientação jurídica - garante a orientação realizada por bacharel em Direito com vistas a dirimir as principais dúvidas no que tange aos aspectos jurídicos de problemas decorrentes da violência sofrida. Realiza encaminhamento para a rede de parceiros os casos que necessitarem de efetivo acompanhamento jurídico de demandas inerentes a condição da vítima; 
d) Pedagogia - realizar visitas às instituições educacionais onde estejam matriculados os menores com objetivo de verificar o desenvolvimento cognitivo e demais questões inerentes à vida escolar, solicitando documentação de histórico escolar para inclusão no processo e elaborar relatório acerca das informações coletadas na unidade escolar, a ser encaminhado ao Psicólogo responsável pelo caso.

\subsection{Atividades Realizadas no Programa}

O programa oferece diversas atividades, dentro do âmbito das três grandes áreas, jurídica, psicológica e assistência social, que segundo o MPAMPGJ (2016) são: a) Oficinas expressivas para crianças - esta atividade ocorre por meio de parcerias com instituições. São atividades realizadas com grupos de crianças, com a orientação de um profissional e que visa por meio da expressão lúdica e artística a promoção da saúde e o fortalecimento dos fatores de proteção da criança, como a resiliência, em um espaço acolhedor e apoiador; b) Grupo de convivência de jovens - esta atividade visa promover a interação social, a discussão grupal a partir de situaçõesproblema, com reflexões que promovam o empoderamento e a construção de identidades; c) Grupo de apoio às mulheres em situação de violência doméstica - esta atividade visa promover a saúde mental das participantes, através da comunicação verbal, da relação terapêutica entre as participantes do grupo e da intervenção profissional, sobretudo por meio de um atendimento acolhedor e humanizado; d) Grupo de convivência de idosos - esta atividade visa a interação social, o fortalecimento da autoestima, favorecer a comunicação e discutir sobre temas relacionados a esse ciclo de vida. Dessa forma, busca-se a potencialização dessas pessoas enquanto sujeitos de direitos; e) Oficinas culturais - esta atividade ocorre por meio de parcerias com instituições com vistas a promover a inclusão cultural e o interesse das pessoas atendidas no programa pelos espaços de cultura da cidade; f) Rodas de conversa - visa discutir e refletir sobre temas diversos, relacionados à cidadania, ao meio ambiente, à promoção da saúde, entre outros; g) Oficinas de geração de renda - esta atividade ocorre por meio de parcerias com demais instituições que desenvolvam atividades de fomento de geração de emprego e renda. Busca desenvolver atividades que favoreçam o empoderamento das pessoas em situação de vulnerabilidade psicossocial por meio de ações que favoreçam o resgate da dignidade humana; h) Grupo de apoio aos familiares -atividade que busca 
contribuir para o restabelecimento de um ambiente familiar favorável à recuperação da dignidade e elevação da autoestima das pessoas em situação de vulnerabilidade psicossocial, bem como de seus familiares, impedindo uma revitimização por parte da própria família. Busca-se oferecer suporte emocional para a superação das perdas provocadas pelo ato ilícito do qual se foi vítima. Além disso, visa criar laços de solidariedade entre os familiares, discutir problemas em comum e promover o apoio no enfrentamento de situações difíceis.

Dentre outras atividades, o programa também realiza visitas domiciliares com o intuito de sensibilizar e conscientizar as famílias sobre a importância do acompanhamento psicossocial nos casos de vulnerabilidade, verificar a realidade da família acompanhada na sua totalidade, fora da instituição e tomar as devidas providências em caso de negligência, insalubridade etc. Inserir a família na rede oferecida pelas políticas públicas nos mais diversos âmbitos para que possa ser dado continuidade ao trabalho realizado na instituição.

\subsection{A importância do Psicólogo no Programa}

O curso de Psicologia no Brasil foi regulamentado em agosto de 1962, logo a clínica se estabeleceu como uma área de atuação do psicólogo, e gradativamente, houve a ascensão de novas modalidades de trabalho, como a da psicologia social e de saúde mental, construindo assim uma nova face para a psicologia (Ferreira Neto, 2010).

A Psicologia trabalha com o intuito de compreender como o indivíduo se manifesta em diferentes contextos, compreendendo a complexidade de comportamentos apresentados, deste modo são realizados no mínimo cinco e a possibilidade de no máximo dez atendimentos psicológicos no programa, e consequentemente são encaminhados para Rede de Atenção à Saúde. A equipe tem conhecimento amplo das características e fatores de riscos associados a situações abusivas, as quais principalmente em crianças que sofrem abusos, que não estão preparadas em nível biológico, psicológico e cultural para tais situações e resultam em danos à saúde, neste sentido os atendimentos psicológicos e avaliações com a equipe de psicólogos não se tornam abusivas para a vítima, e sim um viés de elaboração que minimiza os impactos no desenvolvimento humano. Como forma de atualização e prestação de serviço de qualidade a equipe de psicólogos faz estudos de casos com leituras da área e semanalmente se reúne com a equipe de assistentes sociais para articulação dos processos de trabalho, para otimizar a 
integração e melhorar a qualidade de atendimento dos encaminhados ao programa.

Durante todo o processo é levada em consideração a análise da situação verbal e não verbal e a escuta técnica psicológica é sempre em um ambiente protegido, empático e acolhedor, permitindo a manifestação das emoções e pensamentos buscando dirimir qualquer possibilidade de sentimento de culpa, levando em consideração nos atendimentos a faixa etária da vítima, bem como sua situação emocional no momento, respeitando sempre o direito dos envolvidos.

Nos casos de atendimentos com crianças e adolescentes que sofreram qualquer forma de abusos intrafamiliar, a equipe observou que algumas crianças frequentemente apresentam dificuldades para diferenciar o ato praticado pelo cuidador como sendo abusivo, neste aspecto cabe a equipe nos atendimentos lúdicos e psicoterápicos, diferenciar o abuso de proteção, o papel da família na promoção de suas necessidades básicas, levando em consideração que o abuso sofrido não é culpa da vítima, abrindo assim a possibilidade para uma etapa de desenvolvimento saudável.

A Psicologia tem diferentes teorias e abordagens destacando-se como base no programa algumas abordagens, dentre elas, a psicoterapia breve que é uma intervenção terapêutica com tempo e objetivos limitados. Os objetivos são estabelecidos a partir de uma compreensão diagnóstica do paciente e da delimitação de um foco e esses objetivos passíveis de serem atingidos num espaço de tempo limitado que pode ser, ou não, preestabelecido, através de determinadas estratégias analíticas (Oliveira, 1999).

Outra abordagem utilizada no programa é a terapia cognitiva comportamental, que se utiliza do conceito "biopsicossocial", como compreensão do indivíduo em um olhar holístico, na determinação e compreensão dos fenômenos relativos a psicologia humana, no entanto constitui-se como uma abordagem que foca no trabalho sobre os fatores cognitivos (Saint-Clair \& Navolar, 2004).

Conforme Figueiredo (1996) a clínica psicológica se caracteriza não pelo local em que se realiza, ou seja, o consultório, mas pela qualidade da escuta e do acolhimento que se oferece ao sujeito: a escuta e a acolhida do excluído do discurso.

A terapia familiar sistêmica é uma “[...] técnica de intervenção terapêutica que tem como foco principal a alteração das relações que se passam no sistema familiar, com o objetivo de alívio dos sintomas disfuncionais" (Tondo, 1988, p. 40). Nesta abordagem, representam sistemas abertos em interação com o meio em que estão 
inseridas, estão baseadas em questões econômicas e de propriedade, permeadas por afetos e sentimentos, assumindo as funções de proteção de seus membros, bem como a de transmissão à sua prole de padrões culturais da sociedade da qual fazem parte. (Gabel \& Soares, 2006).

Sendo assim, o setor da psicologia do programa adequa-se à demanda específica apresentada em cada caso, pautando-se na abordagem que melhor se aplica e embasando-se em sua teoria, para o melhor manejo e atendimento dos casos encaminhados.

\section{Relato de Experiência do Estágio no Programa}

O estágio é vital para formação de qualquer profissão, independente da área de atuação, conforme o Art. 20 das Diretrizes Curriculares Nacionais, os estágios obrigatoriamente devem fazer parte da formação, pois representam um conjunto de atividades planejadas e supervisionadas pelos professores da instituição de ensino, articulando entre a teoria durante o curso, com a prática vivenciada no estágio profissional dos estudantes (Brasil, 2011).

As atividades desenvolvidas no estágio variaram entre visitas domiciliares, supervisões e atendimentos realizados nas salas do programa que aconteceram no período de novembro de 2017 a maio de
2018. O enriquecimento da formação dos profissionais em Psicologia envolve, a capacitação de coordenadores de graduação em elaboração de estruturas curriculares baseadas em competências, uma formação baseada na diversificação de métodos e de estratégias na criação de situações de aprendizagem que levem os alunos a demonstrar as competências norteadoras do currículo como solução de problemas e geração de conhecimentos (Cury, 2013).

Os atendimentos são supervisionados pela equipe técnica psicológica que atua no programa composto por três psicólogos, que em discussões sobre o caso orientam da melhor forma como os estagiários podem utilizar os conhecimentos teóricos e técnicos na prática. De acordo com Nunes e Monteiro (2008), por ser um profissional da psicologia, a imagem do supervisor é identificada como importante figura de apoio para as instabilidades das mudanças de papéis.

Os atendimentos são encaminhados à equipe de Psicologia quando é identificada alguma demanda de vulnerabilidade psicossocial, dentre os atendimentos realizados, o objetivo é ressignificar a situação traumática vivida pela vítima ou familiar e amenizar algum tipo de sofrimento que possa afetar o desenvolvimento ou as atividades ocupacionais do assistido. 
Segundo Peres, Mercante e Nasselo (2005) esses tipos de atendimentos, realizados com vítimas de algum trauma psicológico tem como finalidade a atribuição gradual de novos significados às situações que ocorreram no passado, porém, que não ocorrem mais no presente.

Para Piaget (1976) o lúdico é uma rica fonte das atividades intelectuais da criança, quando o atendimento é realizado com menores de 14 anos, utiliza-se a sala de atendimento psicológico infantil, ambiente lúdico onde, através do brincar, a criança projeta o trauma vivenciado durante a sessão e possibilita a identificação de algum tipo de déficit cognitivo ou no desenvolvimento.

Conforme Vigotsky (citado por Wajskop, 2005, p. 32 e 33), “[...] é na brincadeira que a criança se comporta além do comportamento habitual da sua faixa etária, além do seu comportamento diário". A criança vivência uma experiência no brincar como se ela fosse maior do que é na realidade. A brincadeira torna-se então um importante meio pelo qual a criança pode adquirir conhecimentos da realidade que se passa à sua volta.

Quando uma família é acompanhada pelo programa, a equipe se divide para que cada profissional atenda um integrante, e após, possam discutir os casos para assim identificar possíveis demandas a serem trabalhadas em sessão e buscar estratégias para promoção de saúde e inserção na rede de atenção.

Apesar da parte teórica nos proporcionar um conhecimento técnico a vivência do estágio vinculado com a prática é bastante reveladora, todo este processo é habitualmente vivenciado de forma pouco pacífica, devido ser um percurso marcado por momentos de insegurança, frustração, desilusão, questionamentos sobre si próprio e sua "vocação" para a profissão (Caires \& Almeida, 2000). Pois nos primeiros atendimentos, todas essas questões como as angústias, as dúvidas e os medos da atuação são colocadas em xeque. A certeza da formação se dá realmente através das experiências que o estágio nos propiciou, sejam essas experiências de boas ou ruins, de acertos ou erros.

\section{Resultados e Discussões}

A respeito dos conteúdos aprendidos durante o referido período, foi possível agregar conhecimento técnico científico, especialmente no que tange o andamento de um processo jurídico quando há alguma alteração psíquica de uma das partes envolvidas. A convivência com profissionais da Psicologia com experiência na área jurídica possibilitou uma nova visão a respeito da atuação do psicólogo.

Durante o processo de estágio supervisionado foi possível conviver com 
profissionais de perfis diferente, o que auxilia na formação da identidade profissional do estagiário, que ao observar diferentes formas de atuação, acaba de certa forma absorvendo conteúdos de prática com os quais se identifica.

No que tange a experiência de se trabalhar em uma equipe multiprofissional, foi de extrema importância poder observar e vivenciar a dinâmica de diferentes profissionais, que trocam informações concernentes ao caso para elaborar possíveis meios de atingir o jurisdicionado na sua totalidade.

Entretanto, há de se destacar que os profissionais necessitam desenvolver seu papel como integrante de uma equipe, devido haver áreas específicas e nem sempre essa idiossincrasia ser respeitada, ou seja, carece de um amadurecimento, de forma que todos tenham seu valor para que haja uma comunicação horizontal e que cada um seja reconhecido, desempenhando seu papel com excelência, e evitar que conflitos pessoais afetem a dinâmica de trabalho.

Após o término dos atendimentos, é elaborado o relatório psicossocial, que tem como finalidade esclarecer mesmo para quem não seja da área da Psicologia, os promotores e procuradores o máximo de informações possíveis que sejam necessárias para se tomar uma decisão, zelando sempre pelo sigilo profissional e integridade das partes envolvidas nos processos judiciais.

No relatório psicossocial constam informações referentes ao primeiro atendimento, desde condições socioeconômicas até situações relatadas que se referem ao processo judicial, a percepção da equipe durante as visitas e a conclusão. Cabe aos estagiários, buscar os conteúdos para o embasamento teórico dos casos atendidos.

Foi possível perceber que, mesmo sendo um programa desenvolvido no meio jurídico, para subsidiar o trabalho dos promotores, os profissionais da área psicossocial trabalham, visando sempre à pessoa/família atendida. Eles tëm como principal foco de emancipação e empoderamento, para que aqueles que passarem pelo serviço absorvam algo que servirá de subvenção no seu processo de recomeço após a situação traumática que lhe trouxeram até o Programa.

Poder vivenciar a prática do atendimento é uma experiência enriquecedora, alinhar o que se aprende em sala de aula e romper com os muros da vida acadêmica e aplicar os conceitos é vital para uma formação sólida e gratificante.

\section{Considerações Finais}

A prática da Psicologia encontra obstáculos, principalmente aos acadêmicos 
que necessitam vivenciar o processo da experiência em campo, pois cada um possui desconfianças com o que podem se deparar para dar prosseguimento a um processo tão importante durante sua formação, que é o estágio. Apenas estudar as teorias não irá ensinar o graduando a se tornar um profissional, há também a necessidade de um aprendizado mais abrangente que vai além das salas de aulas ou livros, que é prático e sem dúvida uma grande contribuição para a formação.

Nesse sentido, houve momentos que contribuíram para que os estagiários se tornarem profissionais mais técnicos, pautados na teoria, mas com vivência na prática, construindo assim um emaranhado de experiências em todos os âmbitos proporcionados pelo estágio, pois foi possível acompanhar casos que consolidavam os conhecimentos préadquiridos no meio acadêmico.

A experiência oportunizou para que, como acadêmicos do curso de Psicologia, $\mathrm{n}$ [os compreendêssemos a importância da atenção psicossocial às vítimas de violência atendidas pelo programa e por toda a rede, corroborando o conceito de formação crítica e reflexiva.

\section{Referências}

Brasil. Ministério da Educação. Conselho Nacional de Educação. Câmara de Educação Superior. Diretrizes Curriculares Nacionais para os cursos de Graduação em Psicologia. Resolução CNE/CES 5/2011. Diário Oficial da União, Brasília, DF, 16 de março de 2011. Seção 1, p.19.

Caires, S. \& Almeida, L. S. (2000). A experiência de estágio académico: Oportunidades de formação e desenvolvimento do estudante. Psicologia, 14(2), 235-250. Recuperado em 21 de junho de 2018. 2 Disponível em http://www.scielo.mec.pt/scielo.php?script=sci_arttext\&pid=S087420492000000200008\&lng=pt\&tlng=pt.

Cury, B. M. (2013). Reflexões sobre a formação do psicólogo no Brasil: a importância dos estágios curriculares. Psicologia em Revista, 19 (1), 149-151. Recuperado em 20 de junho de 2018, de http://pepsic.bvsalud.org/scielo.php?script=sci_arttext\&pid=S1677$11682013000100012 \& \operatorname{lng}=$ pt\&tlng=pt.

Ferreira Neto, J. L. (2010). Uma genealogia da formação do psicólogo brasileiro. Memorandum, 18, 130-142. Recuperado em 06 de junho de 2018. Disponível em http://www.fafich.ufmg.br/ memorandum/a18/ferreiraneto01.pdf.

Figueiredo, L. C. M. (1996). Revisitando as psicologias. Da epistemologia à ética das práticas e discursos psicológicos. São Paulo / Petropólis: EDUC / Vozes. 
Gabel, C. L. M. \& Soares, H.P. Contribuições da terapia familiar sistêmica para a escolha profissional. Revista Brasileira de Orientação Profissional, 2006, 7 (1), pp. 57 - 64. Recuperado em 18 de junho de 2018. Disponível em: http://pepsic.bvsalud.org/scielo.php?script=sci_arttext\&pid=S1679-33902006000100007.

Ministério Público do Estado do Amazonas (2016). Programa de Atenção às Pessoas em Situação de Vulnerabilidade Psicossocial - RECOMEÇAR. Recuperado em 13 junho, 2018. Disponível em: http://www.mpam.mp.br/programa-recomecar.

Monteiro, F. S. (2012). O papel do psicólogo no atendimento às vítimas e autores de violência doméstica. Monografia, Centro Universitário de Brasília - UniCEUB, Brasília, DF, Brasil.

Monteiro, N. R. Oliveira \& Nunes, M. L.T. (2008). Supervisor de psicologia clínica: um professor idealizado? Psico-USF, 13(2), 287-296.

Oliveira. T. I. (1999). Núcleo de Estudos e Pesquisa em Psicoterapia Breve. Psicologia: Teoria e Prática, Universidade Presbiteriana Mackenzie, 1(2), 9-19.

Peres, J. F. P, Mercante, J. P. \& Nasselo, A. G. (2005). Promovendo resiliência em vítimas de trauma psicológico. Rev Psiquiatr, 27(2), 131-138. doi.org/10.1590/S010181082005000200003

Piaget, J. (1976). Psicologia e Pedagogia. Rio de Janeiro: Forense Universitária.

Saint-Clair, B. \& Navolar, A. B. B. (2004). Terapia Cognitivo-Comportamentais: Conceitos e Pressupostos Teóricos, n. 04, Curitiba, jul. Recuperado em 18 de junho de 2018. Disponível em: www.utp.br/psico.utp.online.

Tondo, C. T. G. (1998). Terapia familiar: Bases, caminhos percorridos e perspectivas. Em Y.S. Souza \& M.L.T. Nunes (Org.) Família, organizações e aprendizagem (pp.37-104). Porto Alegre, RS: PUCRS.

Wayskop, G. (1997). Brincar na pré-escola. São Paulo: Cortez.

\section{Os autores}

Gustavo Souza Zanatta é discente do curso de Psicologia pela Universidade Luterana do Brasil CEULM/ULBRA, e-mail: gugazanatta25@gmail.com

Patricky Batista da Silva é discente do curso de Psicologia pela Universidade Luterana do Brasil CEULM/ULBRA, e-mail: pahtrickypsi@gmail.com

Marcelo Augusto Zacarias é docente e Supervisor em Psicologia pela Universidade Luterana do Brasil CEULM/ULBRA, mestre em Psicologia pela Universidade Federal do Amazonas - UFAM, e-mail: marcelopsi2@gmai.com

Recebido em: 10/12/2018

Aprovado em: 30/12/2018 